\title{
Incidência de Parada Cardíaca durante Anestesia, em Hospital Universitário de Atendimento Terciário. Estudo Prospectivo entre 1996 e 2002 *
}

\section{Cardiac Arrest during Anesthesia at a Tertiary Teaching Hospital. Prospective Survey from 1996 to 2002}

Leandro Gobbo Braz ${ }^{1}$; José Reinaldo Cerqueira Braz, TSA ${ }^{2}$; Norma Sueli Pinheiro Módolo, TSA ${ }^{3}$; Paulo do Nascimento Júnior, TSA ${ }^{3}$; Ana Paula Shuhama ${ }^{4}$; Laís Helena Camacho Navarro ${ }^{1}$

\section{RESUMO}

Braz LG, Braz JRC, Módolo NSP, Nascimento Jr P, Shuhama AP, Navarro LHC - Incidência de Parada Cardíaca durante Anestesia, em Hospital Universitário de Atendimento Terciário. Estudo Prospectivo entre 1996 e 2002

JUSTIFICATIVA E OBJETIVOS: A incidência e causas de parada cardíaca $(P C)$ durante a anestesia variam e são difíceis de comparar diante dos diversos métodos usados nos estudos. A pesquisa teve como objetivo estudar todas as $P C$ ocorridas no intra e pós-operatório, durante um período de sete anos, de 1996 a 2002, em hospital de ensino de atendimento terciário para determinar incidência e causas da PC.

MÉTODO: A incidência prospectiva de PC ocorrida durante a anestesia em 40.941 pacientes consecutivos foi identificada, utilizando-se um Banco de Dados. Todos os casos de PC e óbito foram revisados por uma Comissão, para determinar o fator desencadeante da PC ou óbito. A incidência de $P C$ foi calculada em relação à idade, sexo, estado físico, segundo a classificação da ASA, tipo de atendimento, fatores desencadeantes, como alteração do estado físico do paciente e complicações cirúrgicas e anestésicas, tipo de anestesia e evolução para óbito.

RESULTADOS: Ocorreram 138 PC (33,7:10.000), sendo a maioria em recém-nascidos, crianças até um ano e idosos, no sexo masculino $(65,2 \%)$, em pacientes com estado físico ASA III ou superior, em atendimento de emergência e durante anestesia geral. Alterações do estado físico foram o principal fator de $P C$ (23,9:10.000), seguidas de complicações cirúrgicas isoladamente $(4,64: 10.000)$ ou associadas a alterações do estado físico $(2,44: 10.000)$ e da anestesia isoladamente

${ }^{*}$ Recebido do (Received from) CET/SBA do Departamento de Anestesiologia da Faculdade de Medicina de Botucatu (FMB - UNESP), Botucatu, SP; Prêmio Dr. Renato Ribeiro concedido pela Sociedade Brasileira de Anestesiologia em 2003

1. EX-ME 2 do CET/SBA do Departamento de Anestesiologia da FMB UNESP e atual Pós-Graduando do Curso de Mestrado do Programa de Pós-Graduação em Anestesiologia da FMB, UNESP. Bolsista do CNPq

2. Professor Titular do CET/SBA do FMB - UNESP

3. Professora Adjunta e Livre-Docente do CET/SBA da FMB - UNESP

4. Graduanda $\left(6^{\circ}\right.$ ano) da FMB - UNESP. Bolsa de Iniciação Científica $P I B I C / C N P q$

Apresentado (Submitted) em 05 de janeiro de 2004

Aceito (Accepted) para publicação em 25 de maio de 2004

Endereço para correspondência (Correspondence to)

Dr. José Reinaldo Cerqueira Braz

Departamento de Anestesiologia

Faculdade de Medicina de Botucatu - UNESP

18618-970 Botucatu, SP

E-mail: anestesi@fmb.unesp.br

(c) Sociedade Brasileira de Anestesiologia, 2004
$(1,71: 10.000)$ ou associadas a alterações do estado físico (0,98:10.000). O risco de óbito relacionado à anestesia como fator principal ou contributivo foi igual para ambos (0,49:10.000). As principais causas da mortalidade associada à anestesia foram os problemas ventilatórios $(45,4 \%)$, eventos relacionados à medicação empregada $(27,3 \%)$, aspiração pulmonar $(18,2 \%)$ e hidratação excessiva $(9,1 \%)$.

CONCLUSÕES: A incidência de PC durante a anestesia ainda continua elevada. A maioria das $P C$ e óbitos associados à anestesia foi relacionada ao manuseio das vias aéreas e à administração de medicamentos e anestésicos.

Unitermos: COMPLICAÇÕES: óbito, parada cardíaca; TÉCNICAS ANESTÉSICAS, Geral: peridural, Regional: subaracnóidea

\section{SUMMARY}

Braz LG, Braz JRC, Módolo NSP, Nascimento Jr P, Shuhama AP, Navarro LHC - Cardiac Arrest during Anesthesia at a Tertiary Teaching Hospital. Prospective Survey from 1996 to 2002

BACKGROUND AND OBJECTIVES: Cardiac arrest (CA) incidence and causes during anesthesia are variable and difficult to be compared due design variations of major studies. This survey aimed at evaluating all intra and postoperative CA from 1996 to 2002 at a tertiary teaching hospital to determine CA incidence and causes.

METHODS: The prospective incidence of CA during 40,941 anesthesias was identified from a database. All CA and deaths were reviewed by a Committee in order to determine triggering factors. CA cases were studied as to age, gender, ASA physical status, type of treatment, triggering factors, such as changes in patients physical status and surgical and anesthetic complications, type of anesthesia and evolution to death.

RESULTS: There were 138 CA (33.7:10,000), being most of them neonates, children aged less than 1 year, elderly people, males (65.2\%), physical status ASA III or poorer, in emergency surgeries and during general anesthesia. Physical status changes were the major CA factor $(23.9: 10,000)$ followed by surgical complications alone $(4.64: 10,000)$ or associated to physical status changes $(2.44: 10,000)$ and anesthetic complications alone $(1.71: 10,000)$ or associated to physical status changes $(0.98: 10,000)$. The risk of anesthesia-related death as major or contributing factor was similar for both $(0.49: 10,000)$. Major anesthesia-related death causes were ventilatory problems (45.4\%), drug-related events $(27,3 \%)$, pulmonary aspiration $(18.2 \%)$ and fluid overload $(9.1 \%)$.

CONCLUSIONS: CA incidence during anesthesia is still high. Most anesthesia-related cardiac arrests and deaths were related to airway management and drug and anesthetic administration.

Key Words: COMPLICATIONS: cardiac arrest, death; ANESTHETIC TECHNIQUES, General: epidural, Regional, spinal block 


\section{INTRODUÇÃO}

$E^{\mathrm{n}}$ mbora tenham ocorrido grandes avanços na anestesia e cirurgia, a morbidade e a mortalidade peri-operatórias ainda permanecem importantes, embora pareçam ter diminuído, principalmente a partir da década de 1990. Assim, a maioria dos estudos epidemiológicos na década de 1980 ${ }^{1-13}$ relata incidência de mortalidade associada à anestesia entre 0,7 a 3,7:10.000 anestesias, enquanto os estudos realizados nas décadas de 1990 e $2000{ }^{14-24}$ relatam incidência entre 1 a 2:10.000 anestesias, sendo encontradas incidências abaixo de 1:10.000 em várias estatísticas (Quadro I).

Quadro I - Índice de Mortalidade Associado à Anestesia em Estudos Realizados nas Décadas de 1980 e 1990

\begin{tabular}{lcc}
\hline Autores & $\begin{array}{c}\text { Período de } \\
\text { Referência }\end{array}$ & $\begin{array}{c}\text { Índice de } \\
\text { Mortalidade }\end{array}$ \\
\hline Warden e col. (1994) $^{14}$ & $1984-1990$ & $1: 20.000$ \\
Pedersen (1994) $^{15}$ & $1986-1987$ & $1: 2.500$ \\
Eagle e Davis (1997) $^{16}$ & $1990-1995$ & $1: 40.000$ \\
Cicarelli e col. (1998) $^{17}$ & 1995 & $1: 12.963$ \\
Braz e col. (1999) $^{18}$ & $1985-1995$ & $0,85: 10.000$ \\
Arbous e col. (2001) $^{19}$ & $1995-1997$ & $1,4: 10.0000$ \\
Biboulet e col. (2002) $^{20}$ & $1989-1995$ & $0,6: 10.000$ \\
Newland e col. (2002) $^{21}$ & $1989-1999$ & $0,55: 10.000$ \\
Lagasse (2002) $^{22}$ & $1996-2000$ & $0,79: 10.000$ \\
Chan e Auler Jr JOC (2002) $^{23}$ & $1998-1999$ & $1: 82.641$ \\
Kawashima e col. (2003) $^{24}$ & $1994-1998$ & $1: 10.000$ \\
\hline
\end{tabular}

Os dados epidemiológicos brasileiros são poucos, sendo que Ruiz Neto e col. ${ }^{12}$, em 1986, publicaram estudo retrospectivo sobre a incidência de parada cardíaca durante a anestesia, revisando 51.422 fichas de anestesias realizadas entre 1982 a 1984, em hospital universitário de atendimento terciário. Registraram a incidência de 205 casos de parada cardíaca, ou seja, 39:10.000 anestesias, com 99 óbitos (19:10.000). Se forem computados os óbitos definitivamente causados pela anestesia, encontra-se a taxa de mortalidade de 1,75:10.000 anestesias. Em estudos retrospectivos mais recentes, envolvendo a mesma instituição, encontrou-se maior número de casos de parada cardíaca, ou seja, de 49:10.000 anestesias ${ }^{17}$ e de 51:10.000 anestesias ${ }^{23}$, mas menor taxa de mortalidade associada ao fator anestésico, de $1: 12.963$ anestesias no ano de $1995^{17}$, e de $1: 82.641$ anestesias nos anos de 1998 e $1999^{23}$.

Em nosso meio ${ }^{18}$, verificou-se durante o período de fevereiro de 1988 a março de 1996, a ocorrência de 184 paradas cardíacas durante a anestesia em 58.500 pacientes, ou seja, uma incidência ainda elevada de 31,4:10.000 anestesias, com 124 óbitos (21,7:10.000). Computando-se somente os óbitos associados à anestesia, teve-se a taxa de mortalidade de 0,85:10.000 anestesias.

Estudos realizados em outros países mostraram que a incidência da parada cardíaca durante a anestesia é menor do que em nosso meio, de 1,1 a 30:10.000 anestesias $5,9,10,13,20,21,24,25,30,31$
Considerando-se a importância de estudo epidemiológico sobre a incidência de parada cardíaca durante o ato anestésico-cirúrgico em nosso meio, com a finalidade de proposição de medidas preventivas para aumentar a segurança do ato anestésico-cirúrgico, a pesquisa teve como objetivo estudar a incidência de parada cardíaca nas salas de operação (SO) e de recuperação pós-anestésica (SRPA) em hospital universitário, entre 1996 e 2002.

\section{MÉTODO}

Após autorização de Comissão da Ética Médica, o estudo prospectivo e descritivo foi realizado em 40.941 pacientes consecutivos submetidos a ato anestésico-cirúrgico, no Hospital das Clínicas da Faculdade de Medicina de Botucatu, UNESP, uma instituição pública universitária de atendimento terciário, durante o período de janeiro de 1996 a dezembro de 2002.

Todos os pacientes foram examinados por um anestesiologista, imediatamente antes da realização do ato anestésico-cirúrgico, nas situações de cirurgia de emergência e urgência ou na véspera das cirurgias de rotina. A monitorização básica de segurança na sala de operação (SO) na anestesia regional ou bloqueio peridural e subaracnóideo, além da sedação, incluiu: ECG, pressão arterial não-invasiva e oximetria de pulso. Na anestesia geral, além da monitorização já mencionada, incluiu-se capnografia, concentração de oxigênio e dos anestésicos halogenados ins e expirados, e ventilometria. Na SRPA a monitorização básica utilizada foi à mesma da SO.

O estudo avaliou fichas computadorizadas de Banco de Dados, nas quais foram anotados dados antropométricos, sexo dos pacientes, informações referentes à anestesia e cirurgia, além da morbidade ocorrida em cada paciente na SO e SRPA. Também fizeram parte da avaliação ficha especial para cada parada cardíaca e óbito ocorridos, preenchida logo após a ocorrência do evento; análise do prontuário de cada paciente incluído no estudo e relatório da autópsia, quando realizada. Se necessário, informações complementares foram obtidas em contato com o anestesiologista que participou da anestesia. Com os dados, preparou-se um resumo de cada caso, para que fosse discutido por dois a quatro anestesiologistas do Departamento, com o objetivo de determinar o fator desencadeante da parada cardíaca ou do óbito.

Os casos de parada cardíaca tiveram incidência calculada em relação à idade, sexo, estado físico, segundo a classificação da American Society of Anesthesiologists (ASA) I a V, tipo de atendimento (rotina, urgência ou emergência), clínica cirúrgica, local de ocorrência (SO ou SRPA), fatores desencadeantes (alteração do estado físico do paciente, complicações cirúrgicas e anestésicas), anestesia utilizada e condições de alta da SO ou SRPA (bom, regular ou mau estado geral). Também os fatores que contribuíram para o óbito foram analisados.

A fim de determinar o principal fator desencadeante da parada cardíaca, foram adotadas cinco categorias de causas, Vol. 54, Nº 6, Novembro - Dezembro, 2004 
assim definidas: cirurgia, quando a PC foi decorrente de problemas técnico-cirúrgicos; estado físico, quando da presença de alterações orgânicas que contribuíram decisivamente para a PC; anestesia, quando exclusivamente o ato anestésico foi a causa da PC; e fatores associados a anestesia ou a cirurgia, quando da presença de alterações orgânicas que contribuíram para a PC.

$O$ índice de letalidade foi também determinado para cada fator desencadeante, como a relação entre o número de óbitos decorrentes a fator e o número total de paradas cardíacas. Considerando-se que a ocorrência de parada cardíaca e de óbito é evento raro durante a anestesia, suas incidências foram sempre apresentadas em relação a 10.000 anestesias, como é adotado internacionalmente.

\section{RESULTADOS}

Em 40.941 anestesias realizadas no período de abril de 1996 a dezembro de 2002 , foram computadas 138 paradas cardíacas (33,7:10.000 anestesias). A maioria ocorreu na SO (proporção de 14,3:1 em relação à da SRPA) (Tabela I), envolvendo principalmente recém-nascidos, crianças até um ano, adultos na faixa etária de 51 a 64 anos e idosos (Tabela II); no sexo masculino (proporção de 1,9:1 em relação ao sexo feminino) (Tabela III), em pacientes com estado físico (ASA) igual ou superior a III (Tabela IV), em atendimento de emergência (proporção de 12:1 em relação ao atendimento de rotina) (Tabela $V$ ) e durante a anestesia geral, na proporção de 29,5:1 em relação à anestesia subaracnóidea e peridural (Tabela VI).

Tabela I - Incidência de Parada Cardíaca Segundo o Local de Ocorrência: Sala de Operação (SO) ou Sala de Recuperação Pós-Anestésica (SRPA)

\begin{tabular}{lcccc}
\hline & \multicolumn{4}{c}{ Parada Cardíaca } \\
\hline Total de & $\begin{array}{c}\text { SO } \\
\text { Anestesias }\end{array}$ & $\begin{array}{c}\text { SRPA } \\
\mathrm{n}(\%)\end{array}$ & Total & $\begin{array}{c}\text { Incidência Estimada por } \\
10.000\end{array}$ \\
\hline 40.941 & $\begin{array}{c}129 \\
(93,48 \%)\end{array}$ & $\begin{array}{c}9 \\
(6,52 \%)\end{array}$ & 138 & 33,7 \\
\hline
\end{tabular}

Tabela II - Distribuição da Parada Cardíaca Segundo a Faixa Etária

\begin{tabular}{lcccc}
\hline Faixa Etária & $\begin{array}{c}\text { Total de } \\
\text { Anestesias }\end{array}$ & \multicolumn{3}{c}{ Parada Cardíaca } \\
\hline & & $\mathrm{n}$ & $\%$ & $\begin{array}{c}\text { Incidência Estimada } \\
\text { por 10.000 Anestesias }\end{array}$ \\
\hline$\leq 30$ dias & 696 & 10 & 1,4368 & 143,68 \\
31 dias a $<1$ ano & 1.685 & 11 & 0,6528 & 65,28 \\
1 a $\leq 12$ anos & 6.288 & 8 & 0,1272 & 12,72 \\
13 a $\leq 17$ anos & 2.583 & 4 & 0,1548 & 14,48 \\
18 a $\leq 35$ anos & 11.825 & 18 & 0,1522 & 15,22 \\
36 a $\leq 50$ anos & 7.097 & 23 & 0,3241 & 32,41 \\
51 a $\leq 64$ anos & 5.687 & 34 & 0,5978 & 59,78 \\
$\geq 65$ anos & 5.080 & 30 & 0,5905 & 59,05 \\
\hline
\end{tabular}

Revista Brasileira de Anestesiologia

Vol. 54, Nº 6, Novembro - Dezembro, 2004
Tabela III - Distribuição da Parada Cardíaca Segundo o Sexo

\begin{tabular}{lcccc}
\hline & \multicolumn{4}{c}{ Parada Cardíaca } \\
\hline Sexo & $\begin{array}{c}\text { Total de } \\
\text { Anestesias }\end{array}$ & $\mathrm{n}$ & $\%$ & $\begin{array}{c}\text { Incidência Estimada por } \\
10.000 \text { Anestesias }\end{array}$ \\
\hline Masculino & 19.001 & 90 & 0,4736 & 47,36 \\
Feminino & 21.940 & 48 & 0,2187 & 21,87 \\
\hline
\end{tabular}

Tabela IV - Incidência de Parada Cardíaca Segundo o Estado Físico (ASA) do Paciente

\begin{tabular}{lcccc}
\hline & & \multicolumn{3}{c}{ Parada Cardíaca } \\
\hline Estado Físico & $\begin{array}{c}\text { Total de } \\
\text { Anestesias }\end{array}$ & $n$ & $\%$ & $\begin{array}{c}\text { Incidência Estimada } \\
\text { por 10.000 Anestesias }\end{array}$ \\
\hline ASA I & 19.444 & 3 & 0,0154 & 1,54 \\
ASA II & 13.853 & 8 & 0,0577 & 5,77 \\
ASA III & 5.922 & 33 & 0,5572 & 55,72 \\
ASA IV & 1.509 & 53 & 3,5122 & 351,22 \\
ASA V & 213 & 41 & 19,2488 & $1.924,88$ \\
\hline
\end{tabular}

Tabela V - Incidência de Parada Cardíaca Segundo o Atendimento Realizado

\begin{tabular}{lcccc}
\hline & & \multicolumn{2}{c}{ Parada Cardíaca } \\
\hline Atendimento & $\begin{array}{c}\text { Total de } \\
\text { Anestesias }\end{array}$ & $\mathrm{n}$ & $\%$ & $\begin{array}{c}\text { Incidência Estimada } \\
\text { por 10.000 Anestesias }\end{array}$ \\
\hline Rotina & 22.724 & 46 & 0,2024 & 20,24 \\
Urgência & 15.118 & 17 & 0,1124 & 11,24 \\
Emergência & 3.099 & 75 & 2,4201 & 242,01 \\
\hline
\end{tabular}

Tabela VI - Incidência de Parada Cardíaca Segundo a Anestesia Realizada

\begin{tabular}{lcccc}
\hline & & \multicolumn{2}{c}{ Parada Cardíaca } \\
\hline $\begin{array}{l}\text { Procedimento } \\
\text { Anestésico }\end{array}$ & Total $(\mathrm{n})$ & $\mathrm{n}$ & $\%$ & $\begin{array}{c}\text { Incidência Estimada } \\
\text { por 10.000 Anestesias }\end{array}$ \\
\hline Anestesia geral & 23.658 & 119 & 0,5030 & 50,30 \\
Anestesia raquídea* & 15.052 & 4 & 0,0265 & 2,65 \\
Bloqueio regional $^{\text {Sedação }}$ & 1385 & 0 & 0 & 0,0 \\
Outros $^{* *}$ & 666 & 0 & 0 & 0,0 \\
\hline
\end{tabular}

* Peridural e subaracnóidea

** Anestesia local, cuidados de monitorização e suporte em pacientes ASAV

A distribuição das paradas cardíacas, conforme a especialidade cirúrgica, mostrou maior incidência quando o paciente foi operado por duas ou mais clínicas cirúrgicas (multiclínicas) $(6,67 \%)$, na cirurgia cardíaca $(3,85 \%)$, cirurgia torácica $(1,47 \%)$, cirurgia vascular $(0,71 \%)$ e cirurgia pediátrica $(0,56 \%)$ (Tabela VII).

As alterações do estado físico do paciente foram o fator causal principal de PC, seguidas, em ordem decrescente de incidência, dos seguintes fatores desencadeantes: cirurgia, cirurgia em associação às alterações do estado físico, anestesia isoladamente e anestesia associada às alterações do estado físico (Figura 1). 
Tabela VII - Incidência de Parada Cardíaca nas Clínicas Cirúrgicas

\begin{tabular}{lccc}
\hline Clínica Cirúrgica & $\begin{array}{c}\mathrm{N}^{\circ} \text { de Anestesias } \\
\text { Realizadas no Período }\end{array}$ & \multicolumn{2}{c}{ Parada Cardíaca } \\
\hline Multiclínicas & 105 & $\mathrm{n}$ & $\%$ \\
Cardíaca & 1.013 & 39 & 6,67 \\
Torácica & 886 & 13 & 1,47 \\
Vascular & 1.983 & 14 & 0,71 \\
Pediátrica & 2.500 & 14 & 0,56 \\
Gastrocirurgia & 5.717 & 30 & 0,52 \\
Ortopedia & 4.718 & 10 & 0,21 \\
Neurocirurgia & 2.347 & 4 & 0,17 \\
Urologia & 3.106 & 2 & 0,06 \\
Otorrinolaringologia & 3.260 & 2 & 0,06 \\
Outras & 15.306 & 3 & 0,02 \\
\hline
\end{tabular}

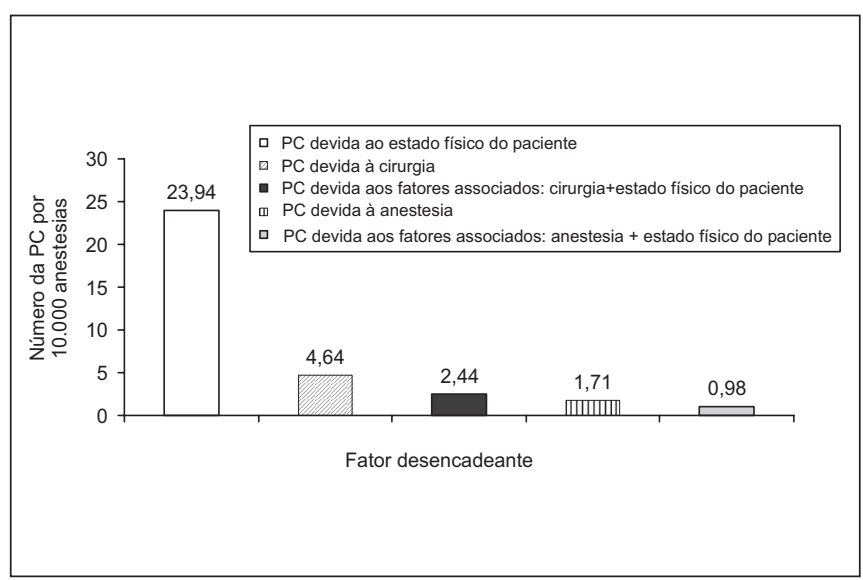

Figura 1 - Incidência de Parada Cardíaca quanto ao Fator Desencadeante

Onze paradas cardíacas foram relacionadas à anestesia (2,69:10.000 anestesias), sendo que sete foram atribuídas exclusivamente à anestesia, resultando em incidência de 1,71:10.000 anestesias, quatro PC tiveram a anestesia como fator contributivo, resultando em incidência de 0,98:10.000 anestesias (Figura 1).

Ocorreram 89 óbitos, com incidência de 21,7:10.000 anestesias, sendo a maioria por alterações do estado físico do paciente. Quatro óbitos foram relacionados à anestesia (0,98:10.000 anestesias), dos quais dois foram atribuídos exclusivamente à anestesia $(0,49: 10.000$ anestesias) e os outros dois tiveram a anestesia como fator contributivo (0,49:10.000 anestesias) (Figura 2).

A letalidade da parada cardíaca que teve a anestesia como fator principal e contributivo foi menor $(36,4 \%)$ em comparação com a causada pelo estado físico do paciente $(70,1 \%)$ ou pela cirurgia $(58,6 \%)$, esta última quando considerada como fator principal e contributivo.

Em relação às condições de alta da SRPA, dos 49 pacientes que apresentaram parada cardíaca sem a ocorrência de óbi-

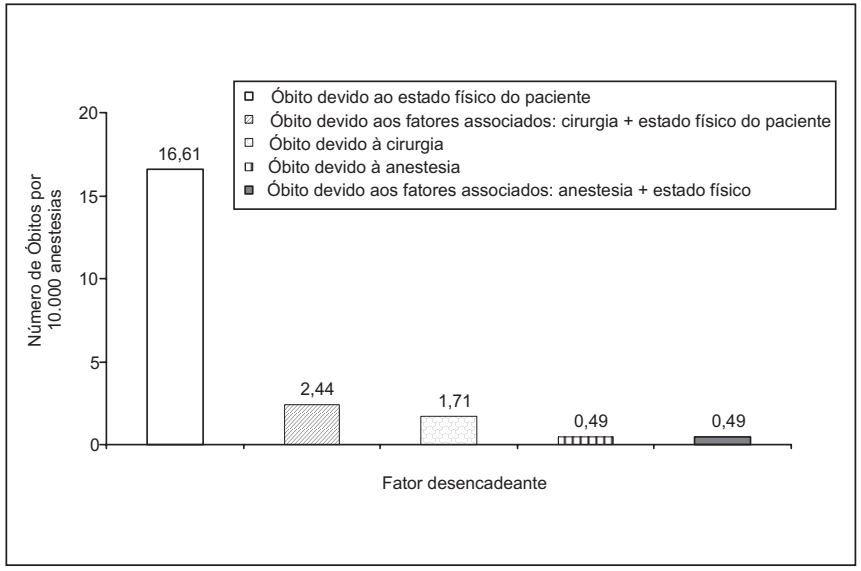

Figura 2 - Incidência de Óbito quanto ao Fator Desencadeante

to, $63,3 \%$ encontravam-se em mau estado geral, $26,7 \%$ em regular estado geral e $10 \%$ em bom estado geral. Em relação às condições de alta dos pacientes que tiveram parada cardíaca por causa anestésica, $36,4 \%$ encontravam-se em mau estado geral e $66,6 \%$, em regular estado geral.

As causas que originaram parada cardíaca associada ou não ao óbito, quando estes tiveram a anestesia como fator desencadeante principal ou associado, estão resumidas na tabela VIII.

Tabela VIII - Causas de Parada Cardíaca e Óbito que tiveram a Anestesia como Fator Desencadeante Principal e Associado com à Alteração do Estado Físico

Ocorrências Freqüência

Aspiração de vômito durante indução de anestesia geral seguida de parada cardíaca e óbito

Aspiração de vômito durante a recuperação de anestesia geral sob intubação traqueal em criança, seguida de hipoxemia, hipoventilação e parada cardíaca

Hipoventilação e hipoxemia imediatamente após extubação traqueal na SRPA seguida de colapso cardiocirculatório e parada cardíaca

Hipoventilação e hipoxemia por extubação acidental durante anestesia geral, seguida de parada cardíaca

Hipóxia por dificuldade de intubação traqueal após a indução da anestesia geral em paciente com insuficiência respiratória grave, seguida de parada cardíaca

Hipóxia por dificuldade de intubação traqueal, após indução de anestesia geral, em paciente obeso, seguida de parada cardíaca e óbito

Colapso cardiocirculatório secundário ao uso de metoprolol durante anestesia geral seguido de parada cardíaca e óbito

Colapso cardiocirculatório seguido de parada cardíaca secundária ao uso de sevoflurano durante anestesia inalatória em criança

Sobrecarga volêmica seguida de edema agudo do pulmão em paciente apresentando oligúria na SRPA, após anestesia geral, seguida de parada cardíaca e óbito

Colapso cardiovascular seguido de parada cardíaca após a indução anestésica em paciente com tamponamento cardíaco 


\section{DISCUSSÃO}

Na literatura, algumas referências abordam o estudo da parada cardíaca durante a anestesia $5,9,10,12,13,20,21,24,26,30,31$, mas a maioria está relacionada à incidência de óbito durante a anestesia ${ }^{1-24}$. Existem grandes dificuldades na comparação entre os vários estudos, em razão das diferenças existentes quanto aos procedimentos cirúrgicos e anestésicos realizados, aos diferentes tipos de pacientes e centros hospitalares estudados e ao período de estudo analisado. Alguns são baseados em declarações voluntárias de incidentes, outros em séries de casos e poucos são prospectivos.

Uma das maiores diferenças entre os estudos de mortalidade diz respeito à definição de morte anestésica. A maioria dos estudos estabelece um período de tempo no qual a morte ocorreu. Variações na definição da mortalidade associada à anestesia podem afetar a determinação da incidência da complicação, como o intervalo de tempo considerado, que pode incluir o período que o paciente permaneceu na SRPA ou se estender a 24 horas ou mesmo a 30 dias após a operação.

No presente estudo, como a definição de mortalidade associada à anestesia limitou-se ao período peri-anestésico e à SRPA, fez-se o estudo da incidência, não apenas, de parada cardíaca, mas também de óbito, ressaltando-se as condições do paciente no momento da alta da SRPA. Deve-se considerar que este estudo foi prospectivo e estes aspectos ampliam e aumentam sua confiabilidade e importância.

Aincidência de parada cardíaca, durante a anestesia e no período de recuperação pós-anestésica, foi de 33,7:10.000 anestesias. Essa incidência é mais alta, em comparação com a de outros estudos internacionais, também recentes, com o de Arbous e col. ${ }^{19}$, que foi de 9:10.000 anestesias, o de Biboulet e col. ${ }^{20}$, de 2,4:10.000, o de Newland e col. ${ }^{21}$, de 19,7:10.000, o de Kawashima e col. ${ }^{24}$, de 7,1:10.000, e o de Sprung e col. ${ }^{25}$, de 4,3:10.000 anestesias. No entanto, é pouco superior à incidência que foi obtida em pesquisa anterior ${ }^{18}$, de 31,4:10.000 anestesias.

Por outro lado, em nosso meio, alguns autores encontraram incidência ainda maior de parada cardíaca, de 39 a $51: 10.000$ procedimentos anestésicos ${ }^{12,17,23}$. Deve-se ressaltar a semelhança existente entre esses estudos e este, pois embora realizados em diferentes décadas, eles foram desenvolvidos em hospitais universitários de atendimento terciário, com prontos socorros atuantes e atendimento à ampla faixa da população e com grande número de cirurgias de urgência e emergência realizadas em pacientes ASA III a $V$, fatos que certamente contribuíram para a elevada incidência de parada cardíaca durante anestesia nesses estudos. Também deve ser considerado que nesses estudos a incidência de parada cardíaca abrangeu o período de até 24 horas após a cirurgia.

Deve-se ressaltar uma característica do método do presente estudo que inclui a experiência de uma única instituição. Esse aspecto, que pode ser considerado negativo, considerando-se que peculiaridades do atendimento podem ter influ- enciado os dados, apresenta, porém, como vantagem a consistência destes, cuja obtenção em estudo multicêntrico dificilmente seria possível. O Hospital das Clínicas da Universidade Estadual Paulista - UNESP é um centro de referência para cuidados terciários, com 400 leitos e 6000 cirurgias realizadas por ano. Ele atende à população estimada de 1.000 .000 de pessoas. Também atende os pacientes vítimas de acidentes automobilísticos de vasta malha rodoviária que envolve auto-estradas. O hospital também é referência para as pacientes obstétricas e recém-nascidos de alto risco, crianças e pacientes portadores de cardiopatias cirúrgicas. Os cuidados anestésicos no hospital são realizados por docentes, médicos e residentes. Assim, a instituição é relativamente pequena, com corpo clínico para o atendimento anestesiológico que pouco se modificou durante o período do estudo.

Ao se considerar a distribuição da parada cardíaca quanto ao fator desencadeante, verificou-se que as alterações do estado físico representaram o principal fator, com incidência de 23,9:10.000, seguido da cirurgia como causa principal ou associada, com incidência de 7,08:10.000 e da anestesia, como causa principal ou associada, com 2,69:10.000 (Figura 1). Em relação à pesquisa anterior ${ }^{18}$, observou-se incidência um pouco menor, de 20:10.000 devido à alteração do estado físico, incidência semelhante de 7,9:10.000 para a causa cirúrgica isolada e associada e incidência maior de 3,6:10.000 como causa anestésica isolada e associada.

No estudo de Ruiz Neto e col. ${ }^{12}$, desenvolvido na década de 1980, a anestesia ocupava o primeiro lugar como causa de parada cardíaca em pacientes submetidos à cirurgia eletiva, na proporção de $76,5 \%$, seguida da cirurgia com $17,6 \%$ e da alteração do estado físico do paciente com 5,9\%. Nas cirurgias de urgência, o ato cirúrgico ocupou o primeiro lugar, com $59,7 \%$, seguido da anestesia com $22,7 \%$ e da alteração do estado físico do paciente com $17 \%$. Assim, comparando-se esses dados com os obtidos na pesquisa, verifica-se que, em nosso meio, houve grande redução do número de paradas cardíacas associadas à anestesia e cirurgia, e, por outro lado, aumento das relacionadas ao estado físico do paciente.

Assim, pelo estudo atual e por outros estudos realizados no país ${ }^{12,17,18,23}$, conclui-se que a incidência de parada cardíaca não está diminuindo em nosso meio, porém suas causas têm-se alterado muito nas últimas décadas, com diminuição, principalmente a partir da década de 1990, das paradas cardíacas de causa anestésica e cirúrgica, mas com aumento considerável das que têm como causa a alteração do estado físico do paciente. O aumento da indicação cirúrgica em pacientes de gravidade progressiva e em idosos (acima de 80 anos), o número crescente de pacientes vitimados por acidentes de trânsito e ferimentos por arma de fogo e branca, nos quais ocorreram quase $20 \%$ das paradas cardíacas computadas no estudo, certamente contribuíram para a elevação do número de paradas cardíacas que tiveram como causa a alteração do estado físico do paciente.

A maior incidência de parada cardíaca durante a anestesia, no sexo masculino (de duas vezes em relação ao sexo femi- 
nino), encontrada na pesquisa (Tabela III), segue a mesma tendência observada em outras pesquisas ${ }^{12,16-23}$. O homem parece estar mais predisposto ao trauma, à violência e à doença vascular do que a mulher, o que pode justificar o resultado obtido.

A ocorrência de parada cardíaca está relacionada ao pior estado físico do paciente (ASA III a V) e ao grau de urgência e principalmente de emergência do procedimento. Assim, a taxa de mortalidade, em pacientes submetidos à cirurgias de emergência é sempre mais elevada do que a observada em cirurgias eletivas $^{7,11,13,16,19,21-23,31,32}$, assim como em pacientes ASA IV ou V ${ }^{11,16,-23,25}$. Observou-se ainda na presente pesquisa, incidência 12 vezes maior de parada cardíaca em cirurgia de emergência do que em relação à cirurgia de rotina (Tabela V) e de 227 vezes maior em pacientes ASAIV do que em pacientes ASA I (Tabela IV).

Nesta pesquisa, o maior número de paradas cardíacas ocorreu nos recém-nascidos, na proporção de 9:1, em relação ao adulto jovem, (18 a 35 anos), na faixa etária das crianças de até 1 ano, na proporção de 4:1, em relação ao adulto jovem, nos pacientes acima de 50 anos, na proporção de $38: 1$, em relação ao adulto jovem; enquanto menor número de paradas cardíacas ocorreu na faixa etária das crianças de 1 a 12 anos, nos adolescentes de 13 a 17 anos e nos adultos jovens, na faixa etária de 18 a 35 anos (Tabela II).

Os estudos demonstraram que pacientes nas faixas etárias extremas são os mais predispostos a sofrer parada cardíaca. Assim, os recém-nascidos e as crianças, principalmente no primeiro ano de vida ${ }^{20,21,23-32}$, apresentam incidência de parada cardíaca durante a anestesia, três a sete vezes maior do que a do adulto jovem ${ }^{11,13,22,23,28,30}$. Nos recém-nascidos e nas crianças até um ano de idade, a ocorrência de prematuridade, doença cardíaca congênita, doença neurológica congênita e outras alterações congênitas parece colocá-los em situação de maior risco anestésico em relação às crianças mais velhas e aos adultos jovens ${ }^{30}$. A idade avançada também aumenta o percentual de ocorrência de parada cardíaca, de 15 a 20 vezes $11,13,18,20,21,25$.

As pesquisas mais recentes realizadas na Austrália ${ }^{16}$, França $^{20}$, Holanda ${ }^{19}$, Estados Unidos ${ }^{25,30}$ e no Brasil ${ }^{23}$, têm verificado a ocorrência de menor número de paradas cardíacase óbitos em crianças de 1 a 12 anos. Entretanto, parece estar aumentando o número de paradas cardíacas e óbitos relacionados à anestesia a partir dos 50 anos, com pico entre $70 \mathrm{e}$ 80 anos, principalmente durante cirurgia de fratura de fêmur e da articulação coxo-femoral ${ }^{16,25,30,32,33}$. Deve-se ressaltar que o número de anestesias realizadas em pacientes de idade igual ou superior a 80 e mesmo 90 anos é cada maior.

Ainda a ser destacado é o fato de que as faixas etárias de 13 a 17 anos e de 18 a 35 anos apresentaram as menores incidências de parada cardíaca, respectivamente de 13 e 15 para 10.000 anestesias. Porém, esta ocorreu, em sua maioria, em jovens do sexo masculino, vítimas de acidente de trânsito ou ferimentos por arma de fogo e branca.

A distribuição da parada cardíaca quanto ao tipo de anestesia mostrou incidência 19 vezes maior para a anestesia geral, em relação aos bloqueios raquídeos (Tabela VI). Outros autores ${ }^{12,18,19,23,25}$ também encontraram resultados semeIhantes. Em comparação com pesquisa anterior ${ }^{18}$, observou-se que a incidência de parada cardíaca durante a anestesia geral permaneceu praticamente a mesma, de 50:10.000 anestesias, enquanto a sua incidência nas anestesias subaracnóidea e peridural diminuiu, passando de 4,4:10.000 anestesias para 2,65:10.000 anestesias. O maior e mais recente estudo prospectivo já realizado mostrou incidência de parada cardíaca durante a anestesia subaracnóidea de 2,7:10.000 anestesias ${ }^{34}$.

Nos dias atuais, com maior conhecimento da fisiologia dos bloqueios espinhais, com o emprego de anestésicos locais mais seguros e com menores efeitos colaterais associados ao aumento da monitorização da oxigenação, por meio da oximetria de pulso, diminuiu, em muito, a possibilidade de ocorrerem complicações importantes durante os bloqueios raquídeos. Entretanto, a parada cardíaca em decorrência de bloqueio espinhal ainda ocorre, embora raramente, como se verificou nesta pesquisa, com incidência de 0,02\% (Tabela $\mathrm{VI}$ ), principalmente em idosos. Felizmente não houve a ocorrência de parada cardíaca relacionada à anestesia como fator desencadeante, em pacientes submetidos a bloqueio raquídeo (Tabela VIII), diferentemente do que ocorreu em estudo anterior ${ }^{18}$, no qual ocorreram cinco paradas cardíacas que tiveram a anestesia raquidiana como fator desencadeante. Na pesquisa mencionada ${ }^{18}$, que foi desenvolvida durante o período de 1988 a 1995, grande parte das anestesias raquidianas não tiveram monitorização pela oximetria de pulso, que somente começou a ser utilizada, de forma rotineira, a partir de 1991. Deve-se considerar que as anestesias peridural e subaracnóidea não são indicadas para pacientes com instabilidade hemodinâmica, fato que pode ter contribuído para a menor incidência de parada cardíaca com esse tipo de anestesia.

Quanto aos bloqueios regionais, a ausência de casos de parada cardíaca já era esperada. As pesquisas têm mostrado que neste tipo de anestesia, por não determinar alterações importantes dos sistemas respiratório e cardiocirculatório, a incidência de parada cardíaca é praticamente nula ${ }^{20}$.

Em relação à especialidade cirúrgica, o maior número de paradas cardíacas ocorreu em pacientes politraumatizados, que foram atendidos, conjuntamente, por várias clínicas, e nas cirurgias cardíacas, torácicas, vasculares e pediátricas. Esses resultados estão de acordo com os obtidos por outros autores ${ }^{17,18,23}$.

Quanto aos óbitos, verificou-se incidência de 21,7:10.000 anestesias, ou seja, praticamente a mesma incidência que se obteve em estudo anterior ${ }^{18}$, que foi de $21,2: 10.000$ anestesias. Esta incidência é menor do que a encontrada em outros estudos nacionais, que varia de 19 a 51:10.000 anestesias 12,17,23. A incidência de parada cardíaca relatada em outros

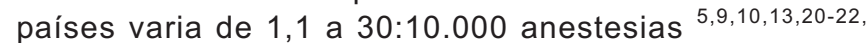
$24,25,28,30,32$

Quanto ao fator desencadeante dos óbitos, verificou-se que a anestesia, de forma isolada e associada a outros fatores, também foi fator menos importante, com incidência de 0,98:10.000 (Figura 2), com menor letalidade, em comparaVol. 54, Nº 6, Novembro - Dezembro, 2004 
ção com os outros fatores desencadeantes. No estudo anterior ${ }^{18}$, verificou-se incidência ligeiramente menor de óbitos, de 0,85:10.000 anestesias.

Em comparação com os resultados obtidos por outros autores, verifica-se que o deste estudo estão dentro da média que tem sido encontrada, principalmente a partir da década de 1990, que é de menos de 1 óbito para 10.000 procedimentos anestésicos.

Desde o estudo pioneiro de Beecher e col. ${ }^{35}$, relativo ao período de 1948-1952, que reportou incidência de óbito de $1: 1560$ anestesias, com a anestesia apresentando fator contributivo importante, e de 1:2680 anestesias, com a anestesia sendo a causa primária da mortalidade, ocorreu redução de, praticamente, 10 vezes da mortalidade de causa anestésica. Mesmo se considerando as diferenças existentes entre o risco dos pacientes e a complexidade das cirurgias realizadas há cinco décadas, conclui-se que, nas últimas décadas houve dramático aumento da segurança durante a anestesia.

Estudo realizado na França mostrou grande aumento do número de procedimentos anestésicos realizados anualmente, de 1980 a 1996, que passou de 6,6 para 13,5 para cada 100 pessoas. Como aspectos importantes observou-se que o maior aumento ocorreu nos idosos e naqueles com estado físico ASA III-V ${ }^{36}$. Caso a tendência seja mundial, ela pode indicar aumento na segurança da Anestesiologia. Esses aspectos fizeram com que os avanços na segurança anestesiológica sejam considerados como modelo, no qual as demais especialidades são encorajadas a estabelecerem estratégias para conseguirem redução similar do risco ${ }^{37,38}$.

Alguns autores ${ }^{22}$ salientaram que a incidência de mortalidade por fator anestésico tem-se mantido estável na última década. Amédia dos estudos realizados nesse período, mostra incidência de 1:13.000 anestesias de óbitos por fator anestésico abrangendo pacientes com estado físico de ASA I a $\mathrm{V}^{22}$. No entanto, essa redução ainda não pode ser considerada como suficientemente boa, pois o ideal é que nenhum paciente morra por causa anestésica, como já referia Macintosh, em $1948^{39}$.

Lagasse ${ }^{22}$, apropriadamente, destaca que a anestesia ainda não é completamente segura para pacientes com estado físico ASA I ou II, nos quais os principais riscos podem ser os iatrogênicos, e muito menos ainda para os pacientes doentes. Infelizmente a anestesia ainda contribui para graves efeitos adversos e mortes evitáveis.

Assim, teria a segurança anestesiológica atingindo um patamar? Os dados obtidos nesta pesquisa e de estudos realizados nas décadas de 1980 e 1990 apontam para essa possibilidade.

Nesta pesquisa, ao se analisarem as causas de parada cardíaca e de óbito, que tiveram a anestesia como fator desencadeante, verificou-se que os problemas ventilatórios ainda se constituem nas causas mais importantes, superando as alterações cardiocirculatórias. Esse aspecto tem sido relatado por vários autores ${ }^{18,23,25-30}$, a despeito da introdução do oxímetro de pulso e da capnografia na prática clínica, que são monitores de segurança do aparelho respiratório.
Outro aspecto a ser destacado é o número de paradas cardíacas e de óbitos relacionados às medicações, fato que ocorreu em três casos de parada cardíaca e foi responsável por dois óbitos. Morray e col. ${ }^{30}$ verificaram que $37 \%$ de todas as paradas cardíacas que ocorreram em crianças estiveram relacionadas à medicação. Já Newland e col. ${ }^{21}$ verificaram em estudo amplo, envolvendo todas as faixas etárias, que $40 \%$ das paradas cardíacas foram relacionadas à anestesia e à medicação empregada. Nenhum dos casos neste estudo relacionados à medicação envolveu o uso errôneo de fármacos, mas a sobredose relativa de anestésicos ou resposta não usual do paciente a uma dose padrão. Em pesquisa anterior ${ }^{18}$, verificaram-se duas paradas cardíacas secundárias ao uso indevido de adrenalina.

Aspecto muito importante a ser destacado é a institucionalização da segurança nos programas das Sociedades e dos Comitês Científicos, criando algoritmos relacionados à monitorização básica, intubação traqueal difícil, reanimação cardiorrespiratória e o treinamento em simuladores. Mas há ainda a necessidade de continuar a perseguir a máxima de que "não existe perigo à vida causado pela anestesia", com a paixão que ela ainda demanda.

Concluindo, a incidência de parada cardíaca durante o ato anestésico-cirúrgico ainda se mantém elevada. O número de paradas cardíacas e de óbitos, que têm como causa a alteração do estado físico do paciente é cada vez mais elevado, enquanto diminui progressivamente o número determinado pela cirurgia e anestesia. Já os óbitos que têm como fator causal a anestesia parece ter atingido um patamar, que não diminuiu na última década. A maior incidência de parada cardíaca ocorre nos recém-nascidos, nas crianças até um ano de idade, nos pacientes acima de 50 anos e nos idosos, no sexo masculino, em pacientes de estado físico ASAIII a V, em cirurgias de emergência, nas que envolvem multiclínicas, e em cirurgias cardíaca, torácica, vascular e infantil.

\section{Cardiac Arrest during Anesthesia at a Tertiary Teaching Hospital. Prospective Survey from 1996 to 2002}

Leandro Gobbo Braz, M.D.; José Reinaldo Cerqueira Braz, TSA, M.D.; Norma Sueli Pinheiro Módolo, TSA, M.D.; Paulo do Nascimento Júnior, TSA, M.D.; Ana Paula Shuhama, M.D.; Laís Helena Camacho Navarro, M.D.

\section{INTRODUCTION}

In spite of major advances in anesthesia and surgery, perioperative morbidity and mortality are still high, although seeming to have decreased, especially as from 1990. So, most epidemiologic studies in the $80 \mathrm{~s}^{1-13}$ report anesthesia-related mortality between 0.7 and $3.7: 10,000$ anesthesias, while studies from the 90 s and 2000 s $^{14-24}$ report 
incidence between 1 to $2: 10,000$ anesthesias, with incidences below 1:10,000 in several statistics (Chart I).

Chart I - Mortality Associated to Anesthesia in Studies from the 80 s and 90 s

\begin{tabular}{lcc}
\hline Authors & Reference Period & Mortality Rate \\
\hline Warden et al. (1994) $^{14}$ & $1984-1990$ & $1: 20,000$ \\
Pedersen (1994) $^{15}$ & $1986-1987$ & $1: 2,500$ \\
Eagle \& Davis (1997) $^{16}$ & $1990-1995$ & $1: 40,000$ \\
Cicarelli et al. (1998) $^{17}$ & 1995 & $1: 12,963$ \\
Braz et al. (1999) $^{18}$ & $1985-1995$ & $0.85: 10,000$ \\
Arbous et al. (2001) $^{19}$ & $1995-1997$ & $1.4: 10,0000$ \\
Biboulet et al. (2002) $^{20}$ & $1989-1995$ & $0.6: 10,000$ \\
Newland et al. (2002) $^{21}$ & $1989-1999$ & $0.55: 10,000$ \\
Lagasse (2002) $^{22}$ & $1996-2000$ & $0.79: 10,000$ \\
Chan \& Auler Jr JOC (2002) $^{23}$ & $1998-1999$ & $1: 82,641$ \\
Kawashima et al. (2003) $^{24}$ & $1994-1998$ & $1: 10,000$ \\
\hline
\end{tabular}

There are few Brazilian epidemiologic data. Ruiz Neto et al. ${ }^{12}$, in 1986, have published a prospective study on the incidence of cardiac arrest during anesthesia after reviewing 51,422 records of anesthesias induced from 1982 to 1984 at a tertiary teaching hospital. They have reported 205 cardiac arrests, that is, 39:10,000 anesthesias, with 99 deaths $(19: 10,000)$. If clearly anesthesia-related deaths are computed, the mortality rate is $1.75: 10,000$ anesthesias. In more recent retrospective studies involving the same institution, a higher number of cardiac arrests has been found, that is, 49:10,000 anesthesias ${ }^{17}$ and $51: 10,000$ anesthesias ${ }^{23}$, but lower anesthesia-related mortality rate has been found in $1995(1: 12,963)^{17}$ and in 1998 and $1999(1: 82,641)^{23}$.

In our institution ${ }^{18}$, in the period February 1988 to March 1996, there have been 184 cardiac arrests during 58,500 anesthesias, that is, a still high incidence of $13.4: 10,000$ anesthesias, with 124 deaths $(21.7: 10,000)$. If only anesthesia-related deaths are computed, there has been mortality rate of $0.85: 10,000$ anesthesias.

Studies in other countries indicate that the incidence of cardiac arrest during anesthesia is lower as compared to Brazil (1.1 to $30: 10,000)^{5,9,10,13,20,21,24,25,30,31}$

Considering the importance of an epidemiologic study on the incidence of cardiac arrest during anesthetic-surgical procedures in Brazil to propose preventive measures to increase anesthetic-surgical safety, our survey aimed at evaluating the incidence of cardiac arrest in operating rooms (OR) and post-anesthetic care units (PACU) at a teaching hospital in the period 1996 to 2002 .

\section{METHODS}

After the Medical Ethics Committee approval, a prospective and descriptive study was performed with 40,941 consecutive patients submitted to anesthetic-surgical procedures at a public tertiary teaching institute (Hospital das Clinicas, Faculdade de Medicina de Botucatu, UNESP) in the period January 1996 to December 2002.
All patients were evaluated by an anesthesiologist immediately before emergency or urgency surgical procedures and the day before routine procedures. Basic safety monitoring in the operating room (OR) during regional anesthesia or epidural and spinal blocks, in addition to sedation, included: ECG, noninvasive blood pressure and pulse oximetry. For general anesthesia, in addition to above-mentioned monitoring, capnography, oxygen and inspired and expired halogenate anesthetics concentration and ventilation parameters were included. Basic PACU monitoring was the same as OR.

The study has evaluated computerized database records from which demographics, gender, anesthesia and surgery-related information and OR and PACU morbidity were extracted. Also part of the evaluation were special records for each cardiac arrest and death, which were filled immediately after the event; evaluation of records of all patients included in the study and of autopsy reports, when performed. If needed, additional information was obtained from the anesthesiologist participating in the procedure. A summary was prepared for each case to be discussed by two to four anesthesiologists of our Department, aiming at determining cardiac arrest or death triggering factor.

Cardiac arrests were calculated based on age, gender, physical status, treatment (routine, urgency or emergency), surgical clinic, place (OR or PACU), triggering factors (changes in physical status, surgical or anesthetic complications), type of anesthesia and OR or PACU discharge conditions (good, regular or poor general status). Factor contributing to death was also evaluated.

To determine major cardiac arrest triggering factor, five categories of causes were adopted: surgery, when CA was a consequence of technical-surgical problems; physical status, in the presence of organic changes decisively contributing to $\mathrm{CA}$; anesthesia, when anesthetic procedure alone was the cause of CA; and associated factors, in the presence of organic changes contributing to $C A$ when added to anesthetic or surgical problems.

Mortality rate was also determined for each triggering factor, such as the relation between the number of deaths caused by the factor and total number of cardiac arrests.

Considering that cardiac arrest and death are uncommon events during anesthesia, their causes were always presented related to 10,000 anesthesias, as internationally adopted.

\section{RESULTS}

From 40,941 anesthesias induced in the period April 1996 to December 2002, there were 138 cardiac arrests (33.7:10,000 anesthesias). Most were observed in the OR (14.3:1 as compared to PACU) (Table I), primarily involving neonates, children below 1 year, adults aged 51 to 64 years and elderly patients (Table II); male patients (1.9:1 as compared to females) (Table III), patients with physical status ASA III or poorer (Table IV), in emergency situations (12:1 as compared to routine procedures) (Table $\mathrm{V}$ ) and during Vol. 54, N 6, Novembro - Dezembro, 2004 
general anesthesia (29.5:1 as compared to spinal and epidural anesthesia (Table VI).

Table I - Incidence of Cardiac Arrest according to Site: Operating Room (OR) or Post-Anesthetic Recovery Unit (PACU)

\begin{tabular}{lcccc}
\hline & \multicolumn{3}{c}{ Cardiac Arrest } \\
\hline $\begin{array}{l}\text { Number of } \\
\text { Anesthesias }\end{array}$ & $\begin{array}{c}\text { OR } \\
\mathrm{n}(\%)\end{array}$ & $\begin{array}{c}\text { PACU } \\
\mathrm{n}(\%)\end{array}$ & Total & $\begin{array}{c}\text { Estimated Incidence by } \\
10,000 \text { Anesthesias }\end{array}$ \\
\hline 40,941 & $\begin{array}{c}129 \\
(93.48 \%)\end{array}$ & $\begin{array}{c}9 \\
(6.52 \%)\end{array}$ & 138 & 33.7 \\
\hline
\end{tabular}

Table II - Cardiac Arrest According to Age

\begin{tabular}{lcccc}
\hline Age Bracket & $\begin{array}{c}\text { Number of } \\
\text { Anesthesias }\end{array}$ & \multicolumn{3}{c}{ Cardiac Arrest } \\
\hline & & $n$ & $\%$ & $\begin{array}{c}\text { Estimated Incidence by } \\
\text { 10,000 Anesthesias }\end{array}$ \\
\hline$\leq 30$ days & 696 & 10 & 1.4368 & 143.68 \\
31 days to $<1$ year & 1,685 & 11 & 0.6528 & 65.28 \\
1 to $\leq 12$ years & 6,288 & 8 & 0.1272 & 12.72 \\
13 to $\leq 17$ years & 2,583 & 4 & 0.1548 & 14.48 \\
18 to $\leq 35$ years & 11,825 & 18 & 0.1522 & 15.22 \\
36 to $\leq 50$ years & 7,097 & 23 & 0.3241 & 32.41 \\
51 to $\leq 64$ years & 5,687 & 34 & 0.5978 & 59.78 \\
$\geq 65$ years & 5,080 & 30 & 0.5905 & 59.05 \\
\hline
\end{tabular}

Table III - Cardiac Arrest by Gender

\begin{tabular}{lcccc}
\hline & & \multicolumn{3}{c}{ Cardiac Arrest } \\
\hline Gender & $\begin{array}{c}\text { Number of } \\
\text { Anesthesias }\end{array}$ & $\mathrm{n}$ & $\%$ & $\begin{array}{c}\text { Estimated Incidence by } \\
\text { 10,000 Anesthesias }\end{array}$ \\
\hline Male & 19,001 & 90 & 0.4736 & 47.36 \\
Female & 21,940 & 48 & 0.2187 & 21.87 \\
\hline
\end{tabular}

Table IV - Cardiac Arrest by Patients' Physical Status

\begin{tabular}{lcccc}
\hline & & \multicolumn{3}{c}{ Cardiac Arrest } \\
\hline $\begin{array}{l}\text { Physical } \\
\text { Status }\end{array}$ & $\begin{array}{c}\text { Number of } \\
\text { Anesthesias }\end{array}$ & $\mathrm{n}$ & $\%$ & $\begin{array}{c}\text { Estimated Incidence by } \\
10,000 \text { Anesthesias }\end{array}$ \\
\hline ASA I & 19,444 & 3 & 0.0154 & 1.54 \\
ASA II & 13,853 & 8 & 0.0577 & 5.77 \\
ASA III & 5,922 & 33 & 0.5572 & 55.72 \\
ASA IV & 1,509 & 53 & 3.5122 & 351.22 \\
ASA V & 213 & 41 & 19.2488 & 1924.88 \\
\hline
\end{tabular}

Table V - Cardiac Arrest by Treatment

\begin{tabular}{lcccc}
\hline Treatment & $\begin{array}{c}\text { Number of } \\
\text { Anesthesias }\end{array}$ & $\mathrm{n}$ & $\%$ & $\begin{array}{c}\text { Estimated Incidence by } \\
10,000 \text { Anesthesias }\end{array}$ \\
\hline Routine & 22,724 & 46 & 0.2024 & 20.24 \\
Urgency & 15,118 & 17 & 0.1124 & 11.24 \\
Emergency & 3,099 & 75 & 2.4201 & 242.01 \\
\hline
\end{tabular}

Revista Brasileira de Anestesiologia

Vol. 54, N 6, Novembro - Dezembro, 2004
Table VI - Cardiac Arrest by Type of Anesthesia

\begin{tabular}{lcccc}
\hline & & \multicolumn{3}{c}{ Cardiac Arrest } \\
\hline $\begin{array}{l}\text { Anesthetic } \\
\text { Procedure }\end{array}$ & Total $(\mathrm{n})$ & $\mathrm{n}$ & $\%$ & $\begin{array}{c}\text { Estimated Incidence } \\
\text { by 10,000 Anesthesias }\end{array}$ \\
\hline General Anesthesia & 23,658 & 119 & 0.5030 & 50.30 \\
Spinal Anesthesia * & 15,052 & 4 & 0.0265 & 2.65 \\
Regional Block & 1,385 & 0 & 0 & 0 \\
Sedation & 666 & 0 & 0 & 0 \\
Others** & 180 & 15 & 8.333 & 833.33 \\
\hline
\end{tabular}

* Epidural and spinal block

** Local anesthesia, monitoring care and support for ASA V patients

The distribution of cardiac arrests according to surgical specialty has shown a higher incidence when patients were operated on by two or more surgical clinics (multiclinics) $(6.67 \%)$, in cardiac surgeries $(3.85 \%)$, chest surgeries $(1.47 \%)$, vascular surgeries $(0.71 \%)$ and pediatric surgeries $(0.56 \%)$ (Table VII).

Table VII - Cardiac Arrest in Surgical Clinics

\begin{tabular}{lccc}
\hline Surgical Clinic & $\begin{array}{c}\text { Number of Anesthesias } \\
\text { in the Period }\end{array}$ & \multicolumn{2}{c}{ Cardiac Arrest } \\
\hline Multiclinics & 105 & $\mathrm{n}$ & $\%$ \\
Heart & 1,013 & 7 & 6.67 \\
Chest & 886 & 39 & 3.85 \\
Vascular & 1,983 & 13 & 1.47 \\
Pediatric & 2,500 & 14 & 0.71 \\
Gastrosurgery & 5,717 & 14 & 0.56 \\
Orthopedics & 4,718 & 30 & 0.52 \\
Neurosurgery & 2,347 & 10 & 0.21 \\
Urology & 3,106 & 4 & 0.17 \\
ENT & 3,260 & 2 & 0.06 \\
Others & 15,306 & 2 & 0.06 \\
\hline
\end{tabular}

Changes in patients' physical status were major CA cause, followed, in decreasing order of incidence, by the following triggering factors: surgery, surgery associated to physical status changes, anesthesia alone and anesthesia associated to physical status changes (Figure 1).

Eleven cardiac arrests were related to anesthesia (2.69:10,000 anesthesias), being 7 exclusively attributed to anesthesia, resulting in an incidence of $1.71: 10,000$ anesthesias. Anesthesia has contributed to $4 \mathrm{CA}$, resulting in an incidence of 0.98:10,000 anesthesias (Figure 1).

There were 89 deaths, with the incidence of $21.7: 10,000$ anesthesias, being most of them related to changes in patients' physical status. Four deaths were attributed to anesthesia (0.98:10,000 anesthesias), being two of them solely attributed to anesthesia (0.49:10,000 anesthesias) and the remaining two had anesthesia as contributing factor (0.49:10,000 anesthesias) (Figure 2).

Cardiac arrest mortality having anesthesia as major or contributing factor has been lower $(36.4 \%)$ as compared to patients' physical status $(70.1 \%)$ or surgery $(58.6 \%)$, the latter considered both major and contributing factor. 
As to PACU discharge conditions, from 49 patients with cardiac arrest and no death, $63.3 \%$ were in poor general status, $26.7 \%$ in regular general status, and $10 \%$ in good general status. As to discharge conditions of patients with anesthesia-related cardiac arrests, $36.4 \%$ were in poor general status and $66.8 \%$ were in regular general status.

Causes of cardiac arrest associated or not to death, when anesthesia was the major or associated triggering factor, are summarized in table VIII.

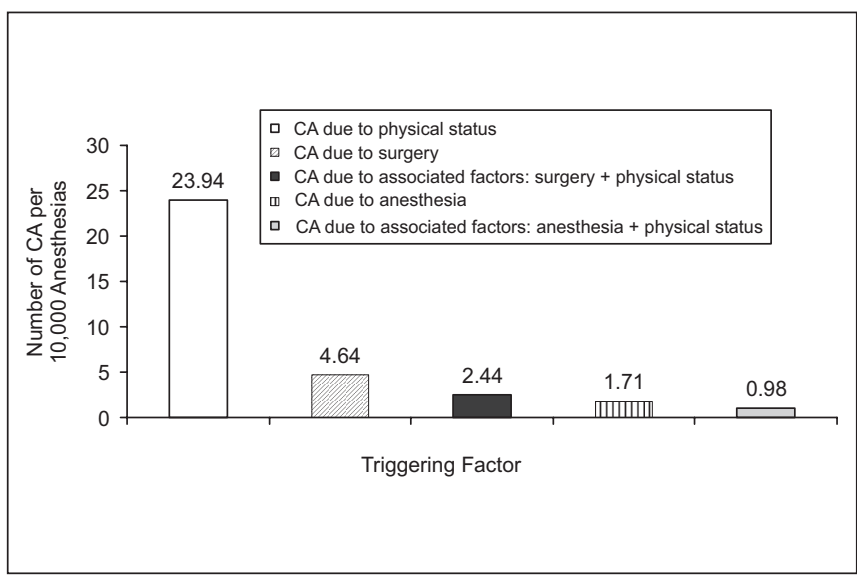

Figure 1 - Cardiac Arrest according to Triggering Factor

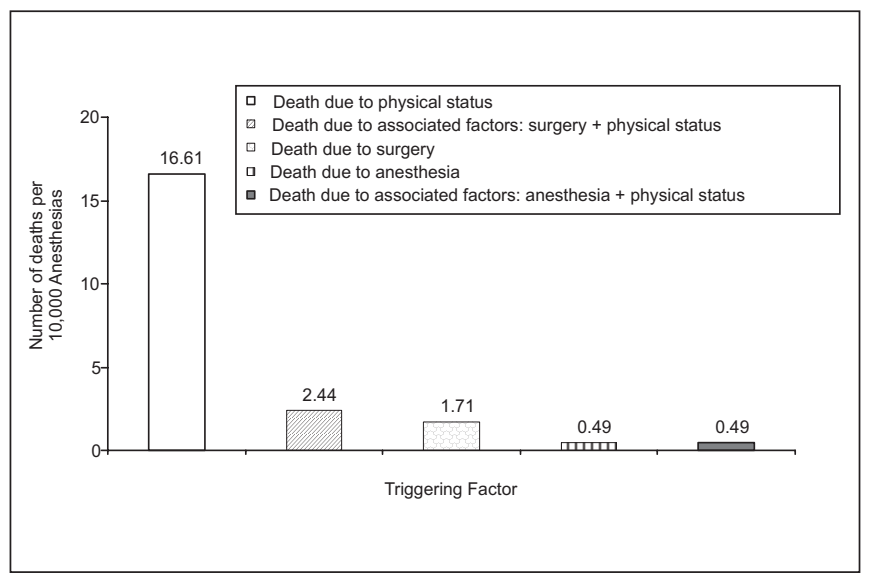

Figure 2 - Deaths According to Triggering Factor
Table VIII - Cardiac Arrest and Deaths caused by Anesthesia as Major Triggering Factor or Associated to Physical Status Changes

\begin{tabular}{|c|c|}
\hline Events & Incidence \\
\hline $\begin{array}{l}\text { Vomit aspiration during general anesthesia induction } \\
\text { followed by cardiac arrest and death }\end{array}$ & 1 \\
\hline $\begin{array}{l}\text { Vomit aspiration during general anesthesia recovery } \\
\text { under tracheal intubation in child, followed by } \\
\text { hypoxemia, hypoventilation and cardiac arrest }\end{array}$ & 1 \\
\hline $\begin{array}{l}\text { Hypoventilation and hypoxemia immediately after tra- } \\
\text { cheal extubation in the PACU, followed by } \\
\text { cardiocirculatory collapse and cardiac arrest }\end{array}$ & 1 \\
\hline $\begin{array}{l}\text { Hypoventilation and hypoxemia by accidental } \\
\text { extubation during general anesthesia, followed by } \\
\text { cardiac arrest. }\end{array}$ & 2 \\
\hline $\begin{array}{l}\text { Hypoxia due to difficult tracheal intubation after gen- } \\
\text { eral anesthesia induction in patient with severe respi- } \\
\text { ratory failure, followed by cardiac arrest }\end{array}$ & 1 \\
\hline $\begin{array}{l}\text { Hypoxia due to difficult tracheal intubation after gen- } \\
\text { eral anesthesia induction, in obese patient, followed } \\
\text { by cardiac arrest and death }\end{array}$ & 1 \\
\hline $\begin{array}{l}\text { Cardiocirculatory collapse secondary to metoprolol } \\
\text { during general anesthesia, followed by cardiac arrest } \\
\text { and death }\end{array}$ & 1 \\
\hline $\begin{array}{l}\text { Cardiocirculatory collapse followed by cardiac arrest } \\
\text { secondary to sevoflurane during inhalational anes- } \\
\text { thesia in child }\end{array}$ & 1 \\
\hline $\begin{array}{l}\text { Fluid overload followed by acute lung edema in pa- } \\
\text { tient with oliguria in the PACU, after general anesthe- } \\
\text { sia, followed by cardiac arrest and death }\end{array}$ & 1 \\
\hline $\begin{array}{l}\text { Cardiovascular collapse followed by cardiac arrest af- } \\
\text { ter anesthetic induction in patient with cardiac patch }\end{array}$ & 1 \\
\hline
\end{tabular}

\section{DISCUSSION}

There are in the literature some references on cardiac arrest during anesthesia $5,9,10,12,13,20,21,24,26,30,31$, but most are related to the incidence of death during anesthesia ${ }^{1-24}$.

There are major difficulties in comparing different studies due to differences in surgical and anesthetic procedures, to different types of patients and hospitals studied, and to the study period. Some are based on voluntary statements of incidents; others on case series, and few are prospective studies.

A major difference among mortality studies is the definition of anesthetic death. Most studies determine a period of time in which there has been death. Variations in the definition of anesthesia-related mortality may affect the computation of the incidence of the complication, such as the time period considered, which may include PACU stay or be extended to 24 hours or even 30 days after surgery.

In our study, since the definition of anesthesia-related mortality was limited to the peri-anesthetic period and PACU stay, we have evaluated the incidence not only of cardiac arrests but also of deaths, highlighting patients' condition at PACU discharge. It has to be considered that ours has been a prospective study and that these aspects enhance and increase its reliability and importance.

The incidence of cardiac arrest during anesthesia and in the post-anesthetic recovery period was $33.7: 10,000$ 
anesthesias. This is a higher incidence as compared to other recent international studies, such as Arbous et al. ${ }^{19}$ who have reported 9:10,000 anesthesias, Biboulet et al. ${ }^{20}$, with 2.4:10,000, Newland et al. ${ }^{21}$, with 19.7:10,000, Kawashima et al. ${ }^{24}$, with $7.1: 10,000$, and Sprung et al. ${ }^{25}$, with 4.3:10,000 anesthesias. However, it is slightly higher than the incidence obtained in a previous survey ${ }^{18}$, which has been $31.4: 10,000$ anesthesias.

On the other hand, some Brazilian authors have found an even higher incidence of cardiac arrest, from 39 to $51: 10,000$ anesthetic procedures ${ }^{12,17,23}$. The similarity of these studies and ours is to be stressed, since although performed in different decades, they were developed in tertiary teaching hospitals, with active first aid units and treating a broad range of the population with a high number of urgency and emergency surgeries performed in ASA III to V patients, factors which certainly have contributed to the high incidence of cardiac arrest during anesthesia in theses studies. It should also be considered that these studies have involved cardiac arrest up to 24 postoperative hours.

One feature of this study was to include the experience of a single institution. This might be considered negative since care peculiarities could have influenced data, but there is also the advantage of data consistency, which would be rather difficult with multicenter study data.

Hospital das Clínicas, Universidade Estadual Paulista UNESP is a reference center for tertiary care, with 400 beds and 6000 surgeries per year, treating an estimated population of 1 million people. It also receives victims of car accidents of a broad highway system. The hospital is also a reference for obstetric patients and high-risk neonates, and for children and patients with surgical heart diseases. Anesthetic care in the hospital is performed by professors, physicians and residents, so the institution is relatively small, with a clinical team for anesthesiologic care that has barely not changed during the study period.

In considering the distribution of cardiac arrest in terms of triggering factors, it has been observed that physical status changes were the major factor, with an incidence of 23.9:10,000, followed by surgery as major or associated cause, with an incidence of 7.08:10,000, and by anesthesia as major or associated cause with 2.69:10,000 (Figure 1). There has been a slightly lower incidence of 20:10,000 as compared to previous survey ${ }^{18}$ due to changes in physical status, similar incidence of 7.9:10,000 for surgery as major or associated cause, and higher incidence of 3.6:10,000 for anesthesia as major or associated cause.

In the study by Ruiz Neto et al. ${ }^{12}$, developed in the 80 s, anesthesia was the major cause of cardiac arrest in patients submitted to elective surgeries, with an incidence of $76.5 \%$, followed by surgery with $17.6 \%$ and physical status changes with $5.9 \%$. For emergency surgeries, surgical procedure was the first with $59.7 \%$, followed by anesthesia with $22.7 \%$ and physical status changes with $17 \%$. So, in comparing these data with our survey, it is observed that in Brazil there has been a major decrease in the number of cardiac arrests asso- ciated to anesthesia and surgery, and an increase in those associated to patients' physical status.

So, according to this study and to other studies performed in our country ${ }^{12,17,18,23}$, one may conclude that the incidence of cardiac arrest is not decreasing in Brazil, but its causes have largely changed in the last decades, with decrease, especially as from the $90 \mathrm{~s}$, of cardiac arrests of anesthetic and surgical origin, but with major increase in those related to physical status changes. The widespread indication of surgeries for patients with progressive severity and elderly (above 80 years), the increasing number of victims of car accidents and gunshot and stab wounds, in whom almost $20 \%$ of cardiac arrests of this study were observed, have certainly contributed to the increased number of cardiac arrests caused by physical status changes.

The higher incidence of cardiac arrests during anesthesia in male patients (twice as compared to females) found in our survey (Table III) follows the same trend of other surveys 12,16-23. Males seem more predisposed to trauma, violence and vascular diseases as compared to females, which may justify our results.

Cardiac arrest is related to poorer physical status (ASA III to $\mathrm{V}$ ) and to the level of urgency and especially emergency of the procedure. So, mortality rates in patients submitted to emergency surgeries are always higher as compared to elective surgeries $^{7,11,13,16,19,21-23,31,32}$, the same being true for ASA IV or $V$ patients ${ }^{11,16,23,25}$. Our study has also observed a 12-fold higher incidence of cardiac arrest in emergency surgeries as compared to routine surgeries (Table V) and 227-fold lower incidence in ASAIV patients as compared to ASAI (Table IV). In our study, the highest number of cardiac arrests was observed in neonates, in the ratio of 9:1 as compared to young adults; in patients above 50 years of age, in the ratio of $38: 1$ as compared to young adults; while the lowest incidence of cardiac arrests was observed in children aged 1 to 12 years, in adolescents aged 13 to 17 years and in young adults, aged 18 to 25 years (Table II).

Studies have shown that patients in extreme age brackets are more predisposed to cardiac arrests. So, neonates and children, especially in their first year of life, the presence of prematurity, of congenital heart disease, of congenital neurological disease and of other congenital disorders, seems to place them at higher anesthetic risk as compared to older children and young adults ${ }^{30}$. Advanced age also increases in 15 to 20 times the risk for cardiac arrest $11,13,18,20,21,25$

Most recent studies in Australia ${ }^{16}$, France $^{20}$, the Netherlands ${ }^{19}$, United States ${ }^{25,30}$ and Brazil ${ }^{23}$, have shown a lower incidence of cardiac arrests and deaths in children aged 1 to 12 years. However, the number of cardiac arrests and deaths related to anesthesia as from 50 years of age seems to be increasing, peaking between 70 and 80 years, especially during hip and hip joint fracture surgery ${ }^{16,25,30,32,33}$. It should be stressed that the number of anesthesias in patients aged 80 or even 90 years is increasingly higher.

It should also be stressed that age brackets from 13 to 17 years and from 18 to 35 years have presented the lowest incidence of cardiac arrest, respectively of 13 and 15:10,000 
anesthesias. These, however, were mostly seen in young males, victims of car accidents or gunshot or stab wounds. Cardiac arrest related to type of anesthesia has shown a 19-fold higher incidence for general anesthesia as compared to spinal blocks (Table VI). Other authors ${ }^{12,18,19,23,25}$ have also found similar results. When comparing with the previous arrest during general anesthesia has remained virtually the same - 50:10,000 anesthesias - while its incidence during spinal and epidural anesthesias has decreased from 4.4:10,000 to $2.65: 10,000$ anesthesias. The largest and most recent prospective study ever performed has shown an incidence of cardiac arrest during spinal anesthesia of $2.7: 10,000$ anesthesias ${ }^{34}$.

Currently, with enhanced knowledge of spinal blocks physiology, with safer local anesthetics with fewer side effects, associated to increased oxygen monitoring through pulse oximetry has highly decreased the possibility of major complications during spinal blocks.

However, spinal block-related cardiac arrest is still seen, although uncommonly as shown by this survey, with incidence of $0.02 \%$ (Table VI), especially in elderly patients. Fortunately, there has been no cardiac arrest related to anesthesia as the triggering factor in patients submitted to spinal blocks (Table VIII), differently from a previous study ${ }^{18}$ where there have been five cardiac arrests with spinal anesthesia as the triggering factor.

In this survey ${ }^{18}$, developed in the period 1988 to 1995 , most spinal anesthesias were not monitored with pulse oximetry, which was only routinely used as from 1991. It should be considered that epidural and spinal blocks are not indicated for patients with hemodynamic instability, fact which might have contributed for the lower incidence of cardiac arrests with this type of anesthesia.

As to regional blocks, the lack of cardiac arrests was to be expected. Studies have shown that in this type of anesthesia, because there are no major respiratory and cardiocirculatory systems changes, cardiac arrest is almost null ${ }^{20}$.

In terms of surgical specialty, there has been a higher number of cardiac arrests in poli-trauma patients jointly treated by several clinics, and in heart, chest, vascular and pediatric surgeries. These results are in line with the literature ${ }^{17,18,23}$.

As to deaths, there has been an incidence of $21.7: 10,000$ anesthesias, that is, virtually the same incidence of a previous study ${ }^{18}$, which has been 21.2:10,000 anesthesias. This incidence is lower than that found by other Brazilian studies, which varies 19 to $51: 10,000$ anesthesias ${ }^{12,17,23}$. The incidence of cardiac arrest reported by other countries varies 1.1 to $30: 10,000$ anesthesias $5,9,10,13,20-22,24,25,28,30,32$.

As to death triggering factors, it has been observed that anesthesia alone or associated to other factors has also been the less important factor, with incidence of 0.98:10,000 (Figure 2) and with lower mortality as compared to other triggering factors. In the previous study ${ }^{18}$, there has been a slightly lower incidence of deaths of $0.85: 10,000$ anesthesias. survey ${ }^{18}$, it has been observed that the incidence of cardiac

As compared to other authors, our results are within the mean found, especially as from the $90 \mathrm{~s}$, which is less than 1 death for 10,000 anesthetic procedures.

Since the pioneer study by Beecher et al. ${ }^{35}$, covering the period 1948-1952, who have reported incidence of death of $1: 1560$ anesthesias when anesthesia was a major contributing factor, and of 1:2680 anesthesias when anesthesia was the primary cause of death, there has been almost a 10-fold decrease in mortality by anesthetic causes. Even when considering differences in patients' risk and complexity of surgeries performed 50 years ago, we have concluded that in the last decades there has been a dramatic increase of anesthetic safety.

Astudy carried out in France has shown major increase in the number of annual anesthetic procedures from 1980 to 1996 , which has gone from 6.6 to 13.5 for every 100 people. As major aspects, it has been observed that the highest increase was among elderly and physical status ASAIII-V patients ${ }^{36}$. If this is a worldwide trend, it may indicate increase in Anesthesiology safety. These aspects have transformed advances in Anesthesiology safety into a model in which other specialties are encouraged to establish strategies to obtain similar risk decrease ${ }^{37,38}$.

Some authors ${ }^{22}$ have stressed that the incidence of mortality by anesthetic factors has been maintained stable in the last decade. The mean of studies performed in this period has shown an incidence of anesthesia-related deaths of 1:13,000 anesthesias involving physical status ASA I to V patients ${ }^{22}$. However, this decrease is still not good enough, because the ideal would be that no patient would die from anesthetic causes, as already stated by Macintosh in $1948{ }^{39}$.

Lagasse ${ }^{22}$, correctly stresses that anesthesia is not yet totally safe for physical status ASA I or II patients, in whom iatrogenic factors may be major risks, and even less for sick patients. Unfortunately anesthesia still contributes for severe adverse events and avoidable deaths.

So, would anesthesia safety have reached a plateau? Data from our survey and studies from the 80 s and 90 s point to this possibility.

In our survey, when analyzing cardiac arrest and death with anesthesia as triggering factors, we have observed that ventilatory problems are still the most important causes, overcoming cardiocirculatory changes. This aspect has been reported by several authors ${ }^{18,23,25-30}$, despite the introduction of pulse oximetry and capnography in the clinical practice, which are safety monitors of the respiratory device. Another aspect to be highlighted is the number of drug-related cardiac arrests and deaths, which has been seen in three cardiac arrests and was responsible for one death. Morray et al. ${ }^{30}$ have shown that $37 \%$ of all cardiac arrests in children were related to medication. Newland et al. ${ }^{21}$ have observed in a broad study involving all age brackets that $40 \%$ of cardiac arrests were related to anesthesia and drugs used. No drug-related case in this study has involved misuse of drugs, but rather anesthetic overdose or unusual response to standard doses. In a previous survey ${ }^{18}$ there have been two cardiac arrests secondary to epinephrine misuse. 
An important aspect to be stressed is the institutionalization of safety programs by Scientific Societies and Committees, creating algorithms related to basic monitoring, difficult tracheal intubation, cardiopulmonary resuscitation and training in simulators. But there is still the need to pursue the principle that "there is no anesthesia-related life threat" with the passion it still demands.

In conclusion, the incidence of cardiac arrests during anesthetic-surgical procedures is still high. The number of cardiac arrests and deaths caused by changes in patients' physical status is increasingly high, while the number determined by surgery and anesthesia is progressively decreasing. Anesthesia-related deaths seem to have reached a plateau, which has not decreased in the last decade. There is a higher incidence of cardiac arrests in neonates, children below 1 year of age, patients above 50 years of age and elderly, in males, in patients physical status ASA III to V, in emergency surgeries, in those involving several clinics and in heart, chest, vascular and pediatric surgeries.

\section{REFERÊNCIAS - REFERENCES}

01. Beecher HK, Todd DP - A study of deaths associated with anesthesia and surgery: based on a study of 559,548 anesthesias in ten institutions 1948-1952, inclusive. Ann Surg, 1954; 140:2-35.

02. Hingson RA, Holden WD, Barnes AC - Mechanisms involved in anesthetic deaths; a survery of operating room and obstetric delivery room related mortality in the University Hospitals of Cleveland, 1945-1955. N Y State J Med, 1956;56:230-235.

03. Clifton BS, Hotten WI - Deaths associated with anaesthesia. Br J Anaesth, 1963;35:250-259.

04. Bodlander FM - Deaths associated with anaesthesia. $\mathrm{Br} \mathrm{J}$ Anaesth, 1975;47:36-40.

05. Minuck M - Cardiac arrest in the operating room - Part I (1965-1974). Can Anaesth Soc J, 1976;23:357-365.

06. Harrison GG - Death attributable to anaesthesia. A 10 - year survey (1967 - 1976). Br J Anaesth, 1978;50:1041-1046.

07 . Hovi-Viander M - Death associated with anesthesia in Finland. Br J Anaesth, 1980;52:483-489.

08. Turnbull KW, Fancourt-Smith PF, Banting GC - Death within 48 hours of anaesthesia at the Vancouver General Hospital. Can Anaesth Soc J, 1980;27:159-163.

09. Lunn JN, Mushin WW - Mortality Associated With Anaesthesia Oxford, Miffield Provincial Hospital Trust, 1982.

10. Keenan RL, Boyan CP - Cardiac arrest due to anesthesia. A study of incidence and causes. JAMA, 1985;253:2373-2377.

11. Tiret L, Desmonts JM, Halton F et al - Complications associated with anaesthesia - a prospective survey in France. Can Anaesth Soc J, 1986;33:336-344.

12. Ruiz Neto PP, Amaral RVG - Parada cardíaca durante a anestesia em um complexo hospitalar. Estudo descritivo. Rev Bras Anestesiol, 1986;36:149-158.

13. Olsson GL, Hallen B - Cardiac arrest during anaesthesia. A computer-aided study in 250,543 anaesthetics. Acta Anaesthesiol Scand, 1988;32:653-664.

14. Warden JC, Borton CL, Horan BF - Mortality associated with anaesthesia in New South Wales, 1984-1990. Med J Aust, 1994;161:585-593.
15. Pedersen $T$ - Complications and death following anaesthesia. A prospective study with special reference to the influence of patient-anaesthesia and surgery-related risk factors. Dan Med Bull, 1994:41:319-331.

16. Eagle CC, Davis NJ - Report of the Anaesthetic Mortality Committee of Western Australia 1990-1995. Anaesth Intensive Care, 1997;25:51-59.

17. Cicarelli DD, Gotardo AOM, Auler Jr JOC et al - Incidência de óbitos anestésicos-cirúrgicos nas primeiras 24 horas. Revisão de prontuários de 1995 no Hospital das Clínicas da FMUSP. Rev Bras Anestesiol, 1998;48:289-294.

18. Braz JRC, Silva ACM, Carlos E et al - Parada cardíaca durante anestesia em Hospital Universitário de atendimento terciário (1988 a 1996). Rev Bras Anestesiol, 1999;49:257-262.

19. Arbous MS, Grobbee DE, van Kleef JW et al - Mortality associated with anaesthesia: a qualitative analysis to identify risk factors. Anaesthesia, 2001;56:1141-1153.

20. Biboulet P, Aubas P, Dubourdieu J et al - Fatal and non fatal cardiac arrests related to anesthesia. Can J Anesth, 2001;48: 326-332.

21. Newland MC, Ellis SJ, Lydiatt CA et al - Anesthetic-related cardiac arrest and its mortality: a report covering 72,959 anesthetics over 10 years from a US teaching hospital. Anesthesiology, 2002;97:108-115.

22. Lagasse RS - Anesthesia safety: model or mith? A review of the published literature and analysis of current original date. Anesthesiology, 2002;97:1609-1617.

23. Chan RPC, Auler Jr JOC - Estudo restrospectivo da incidência de óbitos anestésico-cirúrgicos nas primeiras 24 horas. Revisão de 82.641 anestesias. Rev Bras Anestesiol, 2002:52:719-727.

24. Kawashima Y, Takahashi S, Suzuki M et al - Anesthesia - related mortality and morbidity over a 5-year period in 2,363,038 patients in Japan. Acta Anaesthesiol Scand, 2003;47:809-817.

25. Sprung J, Warner ME, Contreras MG et al - Predictors of survival following cardiac arrest in patients undergoing noncardiac surgery: a study of 518,294 patients at a tertiary referral center. Anesthesiology, 2003;99:259-269.

26. Pottecher T, Tiret L, Desmonts JM et al - Cardiac arrest related to anaesthesia; a prospective survey in France (1978-1982). Eur J Anaesthesiol, 1984;1:305-318.

27. Warden JC, Borton CL, Horan BF - Mortality associated with anaesthesia in New South Wales, 1984 - 1990. Med J Aust, 1994;161:585-593.

28. Cohen MM, Cameron CB, Duncan PG - Pediatric anesthesia morbidity and mortality in the perioperative period. Anesth Analg, 1990;70:160-167.

29. Caplan RA, Posner KL, Ward RJ et al - Adverse respiratory events in anesthesia: a closed claims analysis. Anesthesiology, 1990;72:828-833.

30. Morray JP, Geiduschek JM, Ramamoorthy C et al - Anesthesia-related cardiac arrest in children: initial findings of the Pediatric Perioperative Cardiac Arrest (POCA) Registry. Anesthesiology, 2000;93:6-14.

31. Sigurdsson GH, McAteer E - Morbidity and mortality associated with anaesthesia. Acta Anaesthesiol Scand, 1996;40: 1057-1063.

32. Dupont H, Mezzarobba P, Degremont AC et al - Early perioperative mortality in a multidisciplinary hospital. Ann Fr Anesth Reanim, 1998;17;755-763.

33. Tikkanen J, Hovi-Viander H - Death associated with anaesthesia and surgery in Finland in 1986 compared to 1975. Acta Anaesthesiol Scand, 1995;39:265-267. 
34. Auroy Y, Benhamou D, Bargued L et al - Major complications of regional anesthesia in France: the SOS Regional Anesthesia Hotline Service. Anesthesiology, 2002;97:1274-1280.

35. Beecher HK, Todd DP - A study of the deaths associated with anesthesia and surgery: based on a study of 599,548 anesthesias in ten institutions 1948-1952. Ann Surg, 1954;140:2-35.

36. Clergue F, Auroy $Y$, Pequignot F et al - French survey of anesthesia in 1996. Anesthesiology, 1999;91:1509-1520.

37. Gaba DM - Anaesthesiology as a model for patient safety in health care. BMJ, 2000;320:785-788.

38. Cooper JB, Gaba D - No mith: anesthesia is a model for adressing patient safety. Anesthesiology, 2002;97:1335-1337.

39. Macintosh $\mathrm{R}$ - Deaths under anaesthetics. Br J Anaesth, 1948;21:107-136.

\section{RESUMEN}

Braz LG, Braz JRC, Módolo NSP, Nascimento Jr P, Shuhama AP, Navarro LHC - Incidencia de Parada Cardíaca durante Anestesia, en Hospital Universitario de Servicio Terciario. Estudio Prospectivo entre 1996 y 2002

JUSTIFICATIVA Y OBJETIVOS: La incidencia y causas de parada cardíaca (PC) durante la anestesia varían y son difíciles de comparar delante de los diversos métodos usados en los estudios. El objetivo de la pesquisa fue de como estudiar todas las PC ocurridas en el intra y pos-operatorio, durante un período de siete años, de 1996 a 2002, en un hospital de enseñanza de servicio terciario para determinar incidencia $y$ causas de la PC.
MÉTODO: La incidencia prospectiva de PC ocurrida durante la anestesia en 40.941 pacientes consecutivos fue identificada, utilizándose un Banco de datos. Todos los casos de PC y fallecimiento fueron revisados por una Comisión, para determinar el factor desencadenante de la PC o fallecimiento. La incidencia de la PC fue calculada con relación a la edad, sexo, estado físico, según la clasificación de la ASA, tipo de servicio, factores desencadenantes, como alteración del estado físico del paciente y complicaciones quirúrgicas $y$ anestésicas, tipo de anestesia y evolución para fallecimiento. RESULTADOS: Ocurrieron 138 PC (33,7:10.000), siendo la mayoría en recién nacidos, niños hasta un año de edad y ancianos, en el sexo masculino $(65,2 \%)$, en pacientes con estado físico ASA III o superior, en servicio de emergencia y durante anestesia general. Alteraciones del estado físico fueron el principal factor de PC (23,9:10.000), seguidas de complicaciones quirúrgicas aisladamente $(4,64: 10.000)$ o asociadas a alteraciones del estado físico (2,44:10.000) y de la anestesia aisladamente $(1,71: 10.000)$ o asociadas a alteraciones del estado físico (0,98:10.000). El riesgo de fallecimiento relacionado a la anestesia como factor principal o contributivo fue igual para ambos $(0,49: 10.000)$. Las principales causas de la mortalidad asociada a la anestesia fueron los problemas ventilatorios $(45,4 \%)$, eventos relacionados a la medicación usada (27,3\%), aspiración pulmonar $(18,2 \%)$ e hidratación excesiva $(9,1 \%)$.

CONCLUSIONES: La incidencia de PC durante la anestesia aún continúa elevada. La mayoría de las $P C$ y fallecimientos asociados a la anestesia fue relacionada al manoseo de las vías aéreas y a la administración de medicamentos y anestésicos. 